\title{
Neoadjuvant Chemotherapy in Breast Cancer: A Real-World Practice with Multidisciplinary Team in Southern China
}

\author{
Fang Chen ${ }^{1}$, Lingyu $\mathrm{Ma}^{1}$, Fan $\mathrm{Yi}^{1}$, Manling Zhou ${ }^{1}$, Yaya Liu ${ }^{1}$, Lin Shen ${ }^{1}$, Jian Shi ${ }^{2}$, Haiman Jing ${ }^{1}$, \\ Amy Chang ${ }^{3}$, Ka On Lam ${ }^{1}$ and Hao Yu*1,4 \\ ${ }^{1}$ Department of Clinical Oncology, The University of Hong Kong-Shenzhen Hospital, Shenzhen, China \\ ${ }^{2}$ Department of Breast Surgery, The University of Hong Kong-Shenzhen Hospital, Shenzhen, China \\ ${ }^{3}$ Comprehensive Oncology Center, Hong Kong Sanatorium \& Hospital, Hongkong, China \\ ${ }^{4}$ Shenzhen Institutes of Advanced Technology, Chinese Academy of Sciences, Shenzhen, China \\ *Corresponding author: Hao Yu, Shenzhen Institutes of Advanced Technology, Chinese Academy of Sciences, Shenzhen, Department \\ of Clinical Oncology, The University of Hong Kong-Shenzhen Hospital, Shenzhen, China
}

ARTICLE INFO
Received: 輩 May 20, 2021
Published: 幽 May 28, 2021

Citation: Fang Chen, Lingyu Ma, Fan Yi, Manling Zhou, Hao Yu, et al., Neoadjuvant Chemotherapy in Breast Cancer: A Real-World Practice with Multiple Disciplinary Team in Southern China. Biomed J Sci \& Tech Res 36(1)-2021. BJSTR. MS.ID.005805.

Abbreviations: NACT: Neoadjuvant Chemotherapy; MDT: Multiple Disciplinary Team; S/Es: Side Effects; pCR: Pathological Complete Response; DCR: Disease Control Rate; OS: Overall Survival; EFS: Event-Free Survival; ORR: Objective Response Rate; CR: Complete Response Rate; PR: Partial Response Rate; SD: Stable Disease; PD: Progressive Disease; HER2: Human Epidermal Growth Factor Receptor 2; HR: Hormone Receptor; ddAC*4-P weekly*12: Dose-dense Doxorubicin/ Cyclophosphamide Followed by Weekly Paclitaxel; $\quad \mathrm{AC}^{*} 4-\mathrm{D}^{*} 4$ : Doxorubicin/Cyclophosphamide Followed by Docetaxel; ddAC-P: Dose-Dense Doxorubicin/Cyclophosphamide Followed by Paclitaxel Every 2 Weeks; TCH+/-P: Docetaxel/Carboplatin/Trastuzumab+/-Pertuzumab
ABSTRACT

Background: Neoadjuvant chemotherapy (NACT) is now a standard option in locally advanced breast cancer. However, the level of acceptance varies in different hospitals and there is few study exploring patient's compliance to NACT. This study aimed to evaluate a real-world practice of NACT with Multiple Disciplinary Team (MDT).

Material and Methods: Consecutive patients with locally advanced invasive breast cancers who were advised to receive NACT by MDT between 2014 to 2020 were enrolled in this study. Clinic factors were collected retrospectively, as well as compliance of NACT, reasons of noncompliance and outcomes of treatment. A forest plot was presented to summarize the odds ratios of various factors to predict pathological complete response rate (pCR) by univariate logistic analysis. The EFS was calculated by Kaplan-Meier curve and compared by Log-rank test.

Results: 135 evaluable patients with stage IIA -IIIC breast cancer were recommended of NACT by MDT and 128/135 (94.8\%) patients accepted NACT. Reasons of refusing NACT were mainly worrying about delaying surgery or toxicity of chemotherapy, or patient preference of Chinese traditional medicine. 114/128 (89.1\%) patients had completed all planned NACT and reasons of not completing all cycles were suboptimal clinical response to treatment, and poor patient tolerance to chemotherapy. The total pathological complete response rate (pCR) was $20.3 \%$, and pCR rates were $32.1 \%, 32.6 \%$ and $3.7 \%$ for triple negative, HER-2 positive and HR+/HER-2- subtype respectively. Patients with subtypes of HER2-positive and triple negative breast cancer, ki67 $\geq 30 \%$ had higher pCR $(\mathrm{P}<0.05)$ while age, menopausal status, lymph node involvement and stage had no significant difference. At median follow-up time of $40.0 \mathrm{~m}$, patients with pCR trended to have higher 3 year EFS than those with non-pCR $(92.3 \%$ vs $80.4 \%)$.

Conclusion: There was a high compliance rate of NACT in southern China with the support of MDT. NACT is effective to achieve pCR and satisfactory clinical outcomes, especially for patients with triple negative and HER2-positive breast cancer.

Keywords: Neoadjuvant Chemotherapy; Breast Cancer; Multiple Disciplinary Team 


\section{Introduction}

Neoadjuvant chemotherapy (NACT) is generally recommended for patients with locally advanced breast cancer which are inoperable, or for operable patients who wish to have breastconserving surgery. Based on the current prospective randomized data of 3,946 patients with operable breast cancer, survival rates and disease progression are equivalent for NACT compared to upfront surgery, regardless of histology type [1]. However, the impacts of these two options are different. Some patients may value NACT due to a higher chance of breast conserving surgery rather than mastectomy [2], or make the inoperable advanced tumor become operable [3]. NACT allows a better understanding of tumor response and biology. It can also provide important prognostic information [1,4] and relieve patients' anxiety associated with their cancer $[5,6]$. Patients with pathological complete response (pCR) had higher DCR (disease control rate) and OS (overall survival) benefits, especially those with triple negative or Human epidermal growth factor receptor 2 (HER2)-positive breast cancer [7-10]. One study also found the greater the residual cancer burden, the worse the prognosis [11]. In addition, for those patients who had residual tumor after NACT, it may be helpful to determine whether intensive adjuvant therapy or clinical trials should be performed to improve survivals $[12,13]$. However, if patients can't accept or complete the recommended chemotherapy which can reduce the risk of recurrence and mortality, they can't get any benefits from above evidence either. As we known, compliance with neoadjuvant chemotherapy was carefully monitored and the compliance was usually high in prospective, randomized clinical trials [14-16]. However, there is few study to explore patient's compliance to NACT recommended by physicians in real world practice. The compliance to chemotherapy is suboptimal in real world as one study had showed the acceptance and use of adjuvant chemotherapy was as low as $63 \%$ [17]. Although NACT might improve patient's compliance compared with adjuvant chemotherapy [18], there remains lots of work to be done to improve patient's compliance. It is recommended that NACT should be managed by Multiple Disciplinary Team (MDT). However, this treatment strategy is relatively new in China and level of MDT application and NACT acceptance varies in different hospitals. The current retrospective study aimed to evaluate the compliance and outcomes of NACT in real-world practice with the support of Multidisciplinary Team (MDT) in a hospital of Southern China.

\section{Material and Methods}

\section{Study Population}

Review all 251 chart records of MDT on consecutive patients with breast cancers who were advised to receive NACT between July 2014 to July 2020 in the University of Hong Kong-Shenzhen Hospital. MDT comprises of surgeons, pathologists, radiologists, clinical oncologists and nurses. It is held regularly on Wednesday in this hospital and all patients who plan to receive surgery or have received surgery would be regularly recorded after MDT. Criteria of recommending NACT in the University of Hong Kong-Shenzhen Hospital include: patients with pathology confirmed invasive breast cancer, aged 18-year old and above, tumor size more than $2 \mathrm{~cm}$ (T2 or above) HER2 positive or triple negative breast cancer, clinical axillary node positive or T3/T4 for all disease subtypes. Recurrent or stage IV breast cancer were excluded in this study. During the period of NACT, physical examination was recommended before each cycle of chemotherapy and radiological assessment were arranged at the beginning and end of chemotherapy according to our MDT guideline.

\section{Data Collection}

Following information of patients were retrospectively collected:

1. Patient factors: age, menstrual status

2. Tumor factors: clinical TNM stage, tumor size, ER/PR/ HER2 subtype

3. Treatment factors: NACT regimen and tolerability

4. Reasons of noncompliance and not completing all planned NACT

5. Outcomes of treatment: pathological complete response (pCR) rate and event-free survival (EFS).

\section{Chemotherapy Regimens}

Dose-dense doxorubicin/cyclophosphamide followed by weekly paclitaxel (ddAC*4-Pweekly*12) or doxorubicin/cyclophosphamide followed by docetaxel (AC*4-D*4) is recommended for Hormone receptor (HR)-positive and HER2-negative breast cancer; Dosedense doxorubicin/cyclophosphamide followed by paclitaxel every 2 weeks (ddAC-P) is recommended for triple negative breast cancer; Docetaxel/carboplatin/trastuzumab+/-pertuzumab (TCH+/-P) is advised for HER2-positive breast cancer (Pertuzumab was unavailable in mainland China before 2019.3).

\section{Outcome Measures}

The primary endpoints were compliance of neoadjuvant chemotherapy and pCR. The secondary endpoints were EFS (Eventfree survival) and ORR (Objective response rate). The definition of pCR was absence of residual invasive cancer in the breast and lymph nodes, irrespective of ductal carcinoma in situ (ypT0/is ypN0). EFS was defined as the interval from the beginning of NACT to disease progression, including local progression before surgery or disease recurrence (local, regional, distant) after surgery, or death from any cause. Patients alive without an event as of the analysis cutoff date were censored at last follow-up date. 


\section{Statistical Analysis}

The EFS was calculated by Kaplan-Meier curve, and compared by Log-rank test. We presented a forest plot to summarize the odds ratios of patient, tumor and treatment factors in subgroups to predict pCR by univariate logistic analysis. Cox regression models was performed to estimated hazard ratios (HRs) and 95\% CIs of EFS from stratified with study as a stratification factor. Statistical analysis was performed using R software (version 3.6.2; https:// www.R-project.org). All P values were two-sided and statistical significance was set at $\mathrm{P}<0.05$. All confidence intervals (CIs) were stated at the $95 \%$ confidence level.

\section{Results}

Characteristics of patients, tumors and treatments at baseline A total of 135 patients with local advanced breast cancer were recommended of NACT between July 2014 to July 2020 in the University of Hong Kong-Shenzhen Hospital and 128 patients received NACT (Figure 1). Table 1 list the characteristics of patient, tumor and treatment factors at baseline. The median age of patients was 45 years with majority being premenopausal (72.7\%). Out of the 128 patients who had accepted the recommendation of NACT, all patients $(100 \%)$ had ultrasound scan before and after NACT, $72.4 \%$ patients had mammography before and $73.6 \%$ after NACT. In the meanwhile, $94.6 \%$ patients had breast MR imaging before NACT and $90.7 \%$ after NACT. Compliance of NACT and reasons of noncompliance are shown in Figure 1, out of 135 patients with locally advanced breast cancer deemed necessary for NACT, 5.2\% (7/135) patients refused the recommendation of NACT: $2.2 \%$ (3/135) patients chose to receive Chinese traditional medicine, $3.0 \%(4 / 135)$ patients refused chemo and decided to undergo surgery first due to worry about delaying definitive surgery or toxicity of chemotherapy. All of the 3 patients who chose Chinese traditional medicine have progressed to stage IV diseases and required palliative chemotherapy now. Patients who refused NACT for Chinese traditional medicine and toxicity of chemotherapy had worse EFS compared to those who received NACT (Supplementary Figure 1). 94.8\% (128/135) patients had accepted the recommendation of NACT. Among them, 89.1\% (114/128) of patients completed all NACT and 10.9\% (14/128) of patients accepting the recommendation of NACT could not completing all cycles.

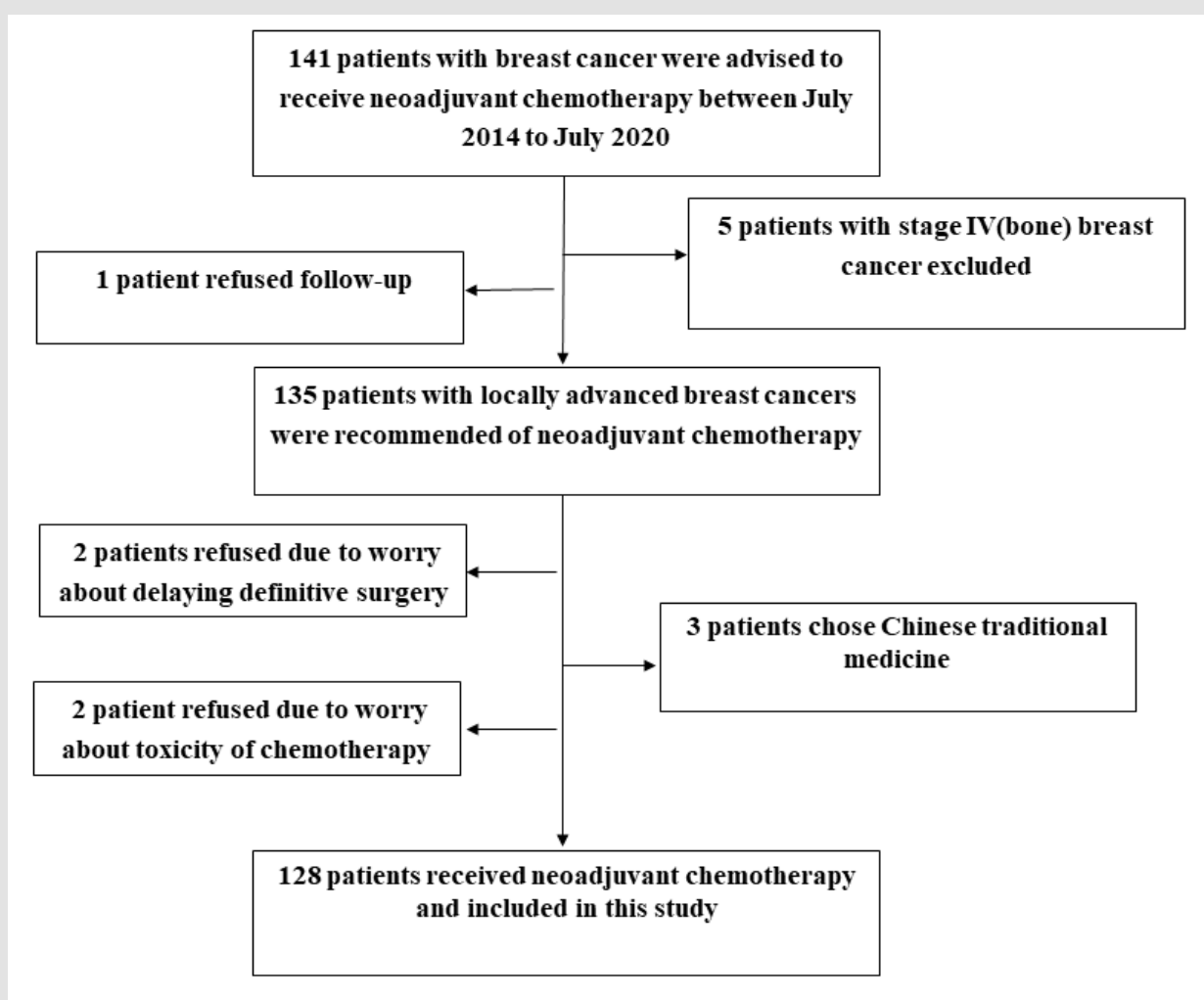

Figure 1: Study population profile 


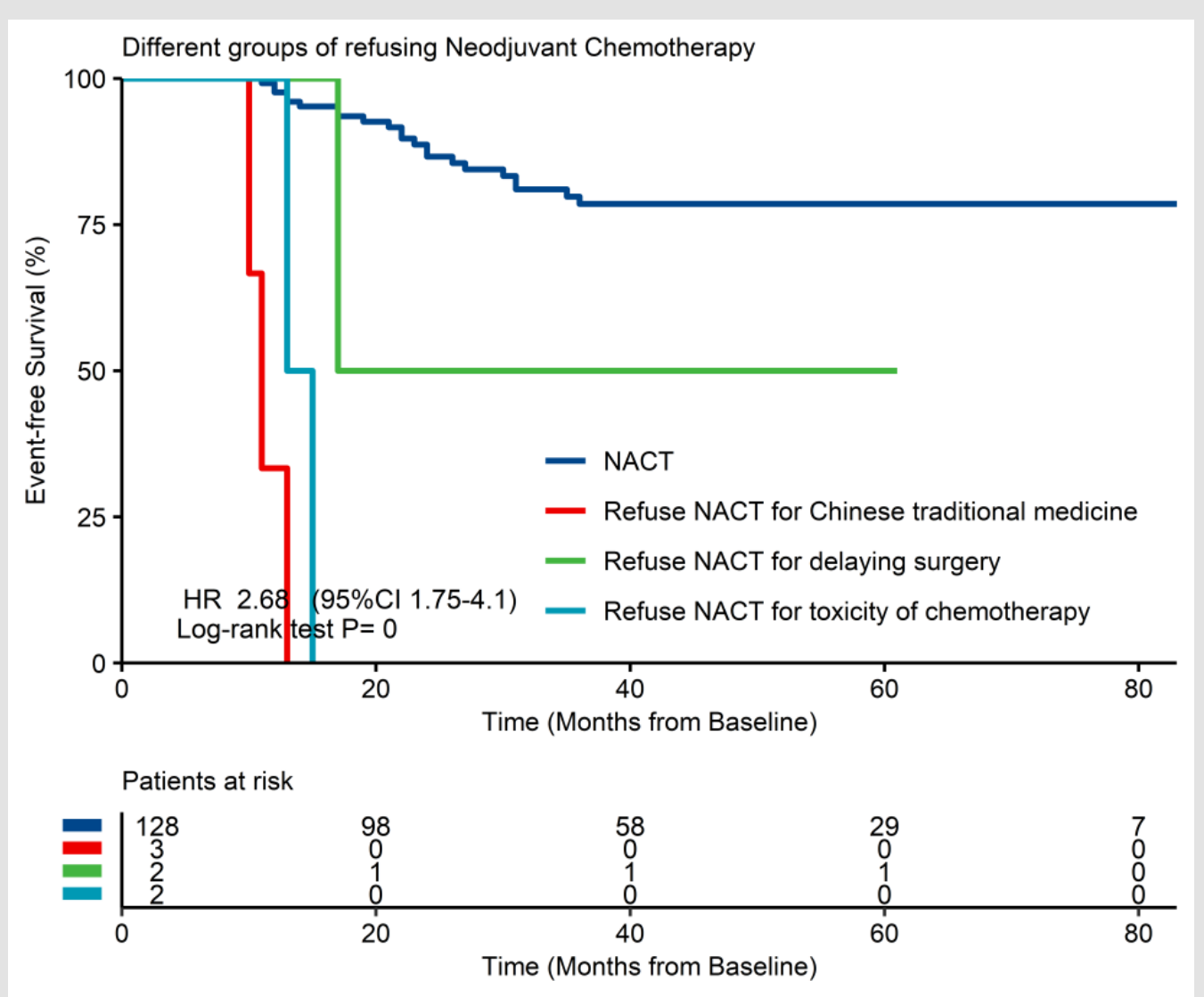

Supplementary Figure 1: Event-free Survival (EFS) grouped by different reasons of refusing Neoadjuvant chemotherapy $(n=135)$.

Table 1: Characteristics of the patient, tumor and treatment factors at baseline.

\begin{tabular}{|c|c|}
\hline & patients $(n=128)$ \\
\hline \multicolumn{2}{|c|}{ Age } \\
\hline Median (range) - year & $45(28-78)$ \\
\hline \multicolumn{2}{|c|}{ Menopausal status - no. (\%) } \\
\hline Premenopausal & $93(72.7 \%)$ \\
\hline postmenopausal & $35(27.3 \%)$ \\
\hline \multicolumn{2}{|c|}{ Tumor laterality } \\
\hline left breast & $68(53.1 \%)$ \\
\hline right breast & $59(46.1 \%)$ \\
\hline bilateral breasts & $1(0.8 \%)$ \\
\hline \multicolumn{2}{|c|}{ Immunohistochemisty subgroup- no. (\%) } \\
\hline HR-positive and HER2-negative & $54(42.2 \%)$ \\
\hline HER2-positive and HR-negative & $21(16.4 \%)$ \\
\hline HER2-positive and HR-positive & $25(19.5 \%)$ \\
\hline Triple negative & $28(21.9 \%)$ \\
\hline \multicolumn{2}{|c|}{ Clinic N stage- no. (\%) } \\
\hline No & $21(16.4 \%)$ \\
\hline $\mathrm{N} 1$ & $62(48.4 \%)$ \\
\hline $\mathrm{N} 2$ & $29(22.7 \%)$ \\
\hline N3 & $16(12.5 \%)$ \\
\hline
\end{tabular}




\begin{tabular}{|c|c|}
\hline \multicolumn{2}{|c|}{ Clinic stage- no. (\%) } \\
\hline IIA & $21(16.4 \%)$ \\
\hline IIB & $43(33.6 \%)$ \\
\hline IIIA & $32(25.0 \%)$ \\
\hline IIIB & $16(12.5 \%)$ \\
\hline IIIC & $16(12.5 \%)$ \\
\hline & Planned chemotherapy regimens- no. (\%) \\
\hline ddAC-P & $30(23.4 \%)$ \\
\hline ddAC-P weekly & $44(34.4 \%)$ \\
\hline AC-D & $8(6.3 \%)$ \\
\hline TCH & $35(27.3 \%)$ \\
\hline TCHP & $11(8.6 \%)$ \\
\hline
\end{tabular}

ddAC-P: Dose-dense doxorubicin/cyclophosphamide followed by paclitaxel every 2 weeks; ddAC*4-P weekly:

Dose-dense doxorubicin/cyclophosphamide*4 cycles followed by weekly paclitaxel *12 cycles; AC-D:

Doxorubicin/cyclophosphamide followed by docetaxel; TCH: Docetaxel/carboplatin/trastuzumab; TCHP:

Docetaxel/carboplatin/trastuzumab/pertuzumab.

Reasons of not completing planned NACT were in 3 categories (Figure 2): response to treatment, side effects (S/Es) and patient preference: 7 due to suboptimal clinical response from chemotherapy with 3 of them having disease progression during chemotherapy; 6 due to S/Es with 2 patients of grade 3 (G3) liver function derangement, 1 of G3 severe pneumonia, 2 of G3 drug allergy and 1 of G3 gastrointestinal (GI) adverse reaction; 1 patient decided to undergo surgery earlier.

\section{Treatment outcomes of neoadjuvant chemotherapy}

As Figure 3 showed, total pathological complete response (tpCR) rate of these 128 patients was 20.3\%. Pathology CR rate was $32.1 \%, 32.6 \%$ and $3.7 \%$ for triple negative, HER2-positive and
HR-positive/HER2-negative subtype respectively. Among the 46 patients with HER2-positive breast cancer, pCR rate of HR-negative subgroup was the highest $(47.6 \%)$ with all patients were HER2 IHC 3 score. As Figure 4 showed, subtype and ki67 were associated with higher pCR. Patients with subtypes of HER2-positive and triple negative breast cancer, ki67 $\geq 30 \%$ had higher pCR $(\mathrm{P}<0.05)$ while age, menopausal status, lymph node involvement, tumor and clinic stage had no significant difference. The median follow-up time was $40.0 \mathrm{~m}$ (95\%CI: 39.0, 46.5). As Figure 5 showed, 3 year EFS was better in pCR group than in non-pCR group $192.3 \%$ vs $80.4 \%$ ) although there was no significant difference which may due to small study population and inadequate follow-up time.

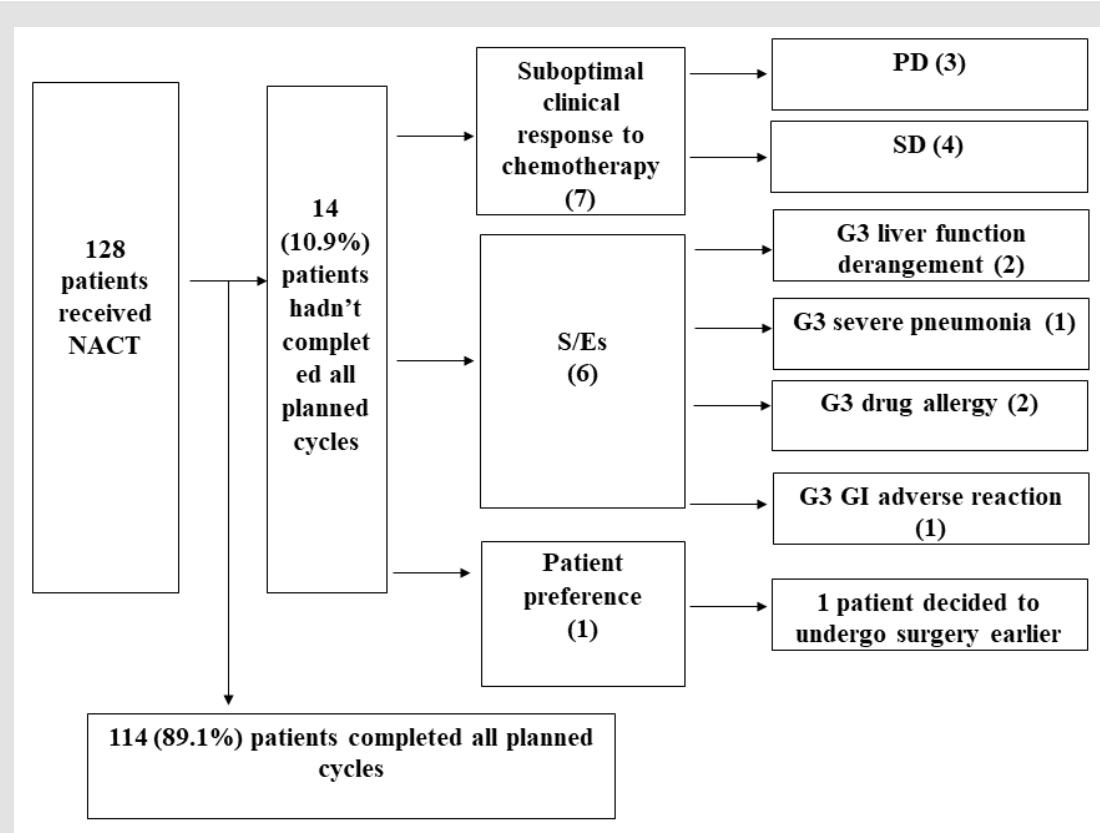

Figure 2: Reasons of not completing all planned cycles of NACT.

S/Es: side effects; PD: progressive disease; SD: stable disease; G3: grade 3; GI: gastrointestinal. 


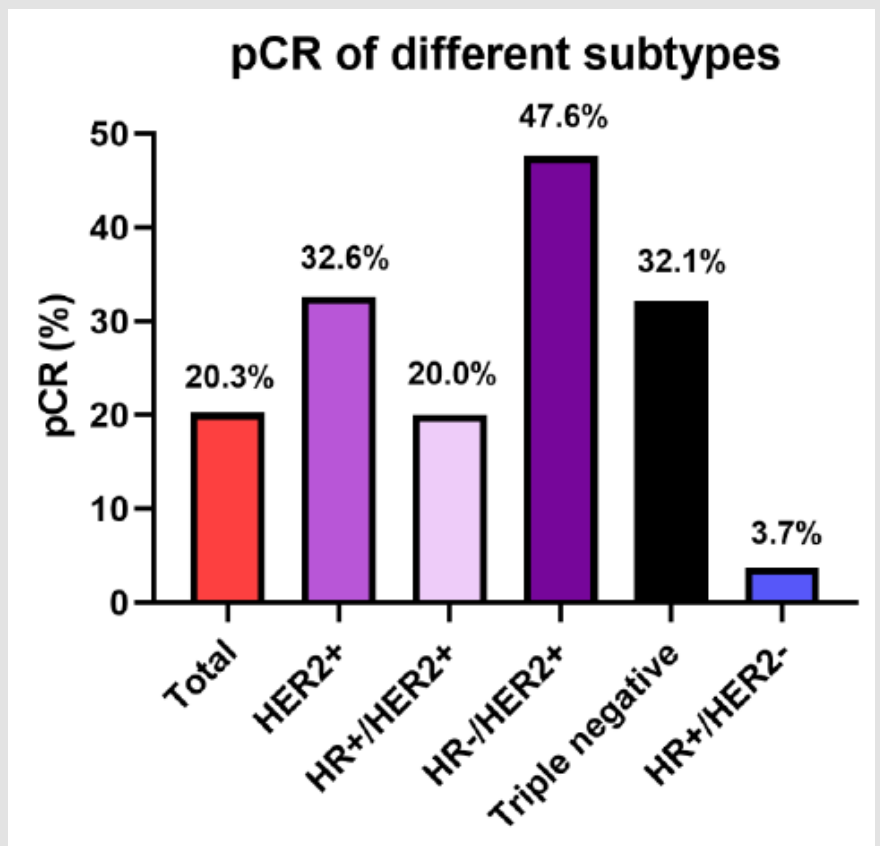

Figure 3: Pathology CR rate of different subtypes

\begin{tabular}{|c|c|c|c|c|}
\hline & & $\mathrm{n}(\%)$ & OR $(95 \% \mathrm{Cl})$ & $\mathrm{P}$ Value \\
\hline Age & 中 & $128(100 \%)$ & $1(0.96,1.04)$ & 0.91 \\
\hline $\begin{array}{l}\text { Menopausal status } \\
\text { Premenopausal } \\
\text { Postmenopausal }\end{array}$ & H & $\begin{array}{l}93(72.7 \%) \\
35(27.3 \%)\end{array}$ & $\begin{array}{c}1 \\
0.97(0.37,2.57)\end{array}$ & 0.96 \\
\hline $\begin{array}{l}\text { Hormone receptor status } \\
\text { ER-negative and PR-negative } \\
\text { ER-positive and/or PR-positive }\end{array}$ & 4 & $\begin{array}{l}49(38.3 \%) \\
79(61.7 \%)\end{array}$ & $0.15(0.06,0.4)$ & $<0.001$ \\
\hline $\begin{array}{l}\text { HER2 status } \\
\text { HER2 negative } \\
\text { HER2 positive }\end{array}$ & $1=-1$ & $\begin{array}{l}82(64.1 \%) \\
46(35.9 \%)\end{array}$ & $3.12(1.29,7.57)$ & 0.01 \\
\hline $\begin{array}{l}\text { Subgroup } \\
\text { HR-positive and HER2-negative } \\
\text { HER2-positive and HR-negative } \\
\text { HER2-positive and HR-positive } \\
\text { Triple negative }\end{array}$ & $\stackrel{\longmapsto}{\longmapsto}$ & $\begin{array}{l}54(42.2 \%) \\
21(16.4 \%) \\
25(19.5 \%) \\
28(21.9 \%)\end{array}$ & $\begin{array}{c}1 \\
23.64(4.53,123.28) \\
6.50(1.17,36.26) \\
12.32(2.44,62.22)\end{array}$ & $\begin{array}{c}<0.001 \\
0.03 \\
0.002\end{array}$ \\
\hline $\begin{array}{l}\text { ki } 67 \\
\text { Ki67 }<30 \% \\
\text { Ki67 } \geq 30 \%\end{array}$ & $\mapsto$ & $\begin{array}{l}31(24.2 \%) \\
97(75.8 \%)\end{array}$ & $4.77(1.06,21.48)$ & 0.04 \\
\hline $\begin{array}{l}\text { Tumor stage } \\
\text { T1 } \\
\text { T2 } \\
\text { T3 } \\
\text { T4 }\end{array}$ & + & $\begin{array}{c}8(6.3 \%) \\
79(61.7 \%) \\
21(16.4 \%) \\
20(15.6 \%)\end{array}$ & $\begin{array}{c}1 \\
2.37(0.27,20.49) \\
0.74(0.06,9.46) \\
1.24(0.11,14.01)\end{array}$ & $\begin{array}{l}0.43 \\
0.81 \\
0.86\end{array}$ \\
\hline $\begin{array}{l}\text { Lymph node involvenment } \\
\text { Negtive } \\
\text { Positive }\end{array}$ & $H$ & $\begin{array}{c}21(16.4) \\
107(83.6 \%)\end{array}$ & $0.78(0.26,2.38)$ & 0.66 \\
\hline $\begin{array}{l}\text { Stage } \\
\text { 川A//IB } \\
\text { IIIA/IIIB/IIC }\end{array}$ & 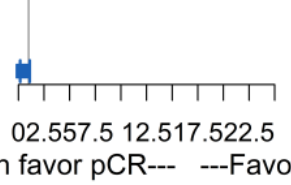 & $\begin{array}{l}64(50.0 \%) \\
64(50.0 \%)\end{array}$ & $0.45(0.18,1.11)$ & 0.08 \\
\hline
\end{tabular}

Figure 4: Subgroup analysis of pCR 


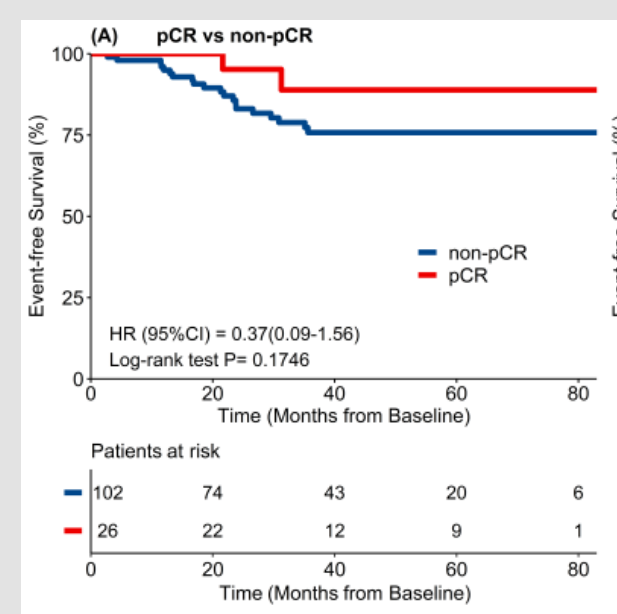

(D) Hormone-receptor-negative
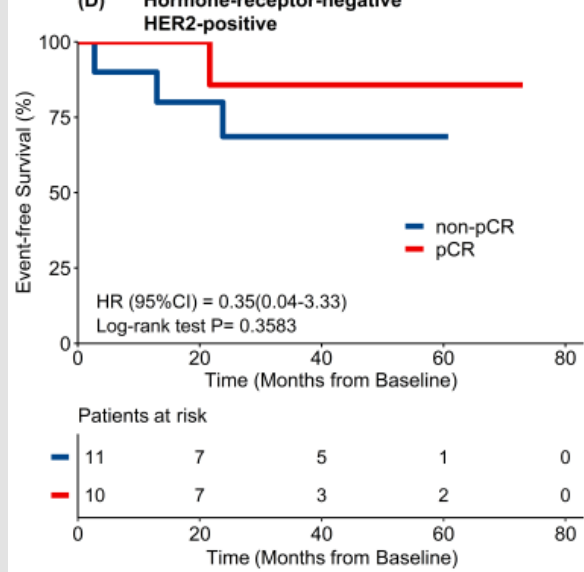
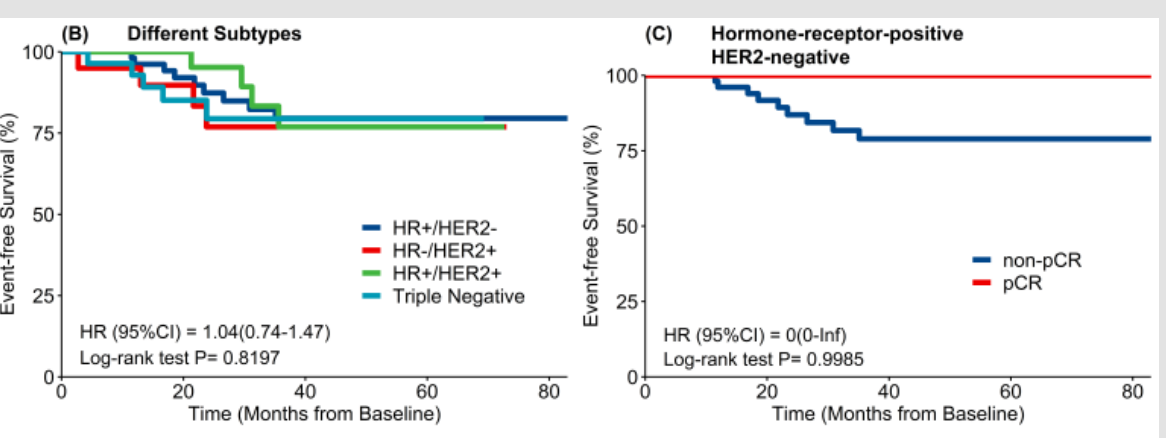

Patients at risk

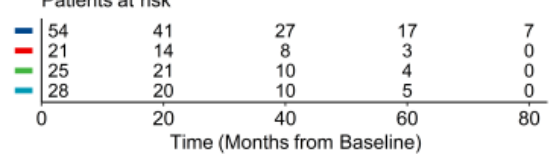

(E) Hormone-receptor-positive

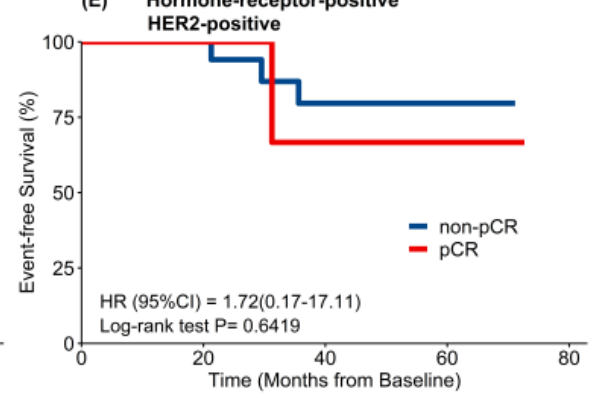

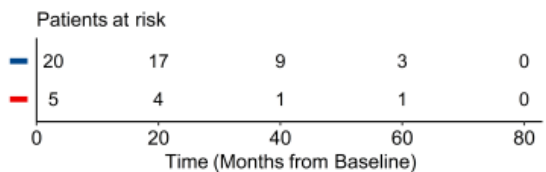
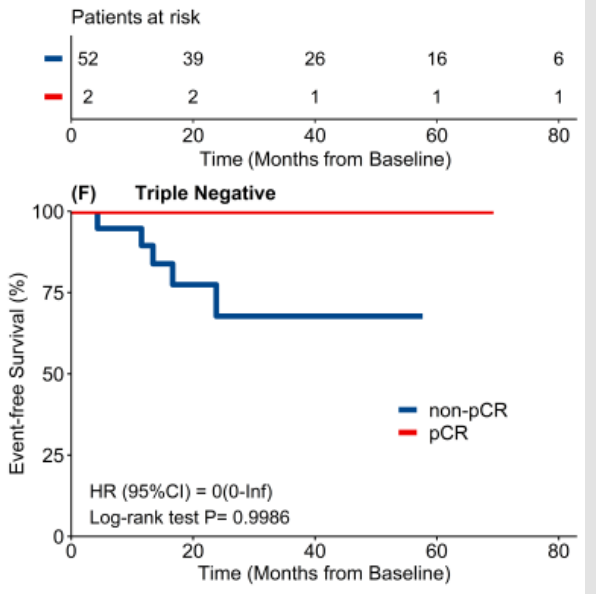

Patients at risk
-\begin{tabular}{ccccc}
19 & 11 & 3 & 0 & 0 \\
- & 9 & 7 & 5 & 0 \\
\hline 0 & 20 & 40 & 60 & 80
\end{tabular}

Figure 5: Event-free survival (EFS)

The EFS benefits can also be observed in all subtypes excepted for HR-positive and HER2-positive patients which probably due to small study sample. As Table 2 showed, total objective responses rate (ORR) of 128 patients was 87.5\% (112/128), and ORR was 82.1\%, 91.3\% and $87.0 \%$ for triple negative, HER2-positive and HR-positive /HER2- negative subtype respectively. 3.1\% (4/128) patients progressed after NACT (most of them were special

Table 2: Objective responses rate (ORR) of neoadjuvant chemotherapy ( $n=128)$.

\begin{tabular}{|c|c|c|c|c|c|c|}
\hline & Total $(n=128)$ & $\begin{array}{l}\text { triple negative } \\
\qquad(n=28)\end{array}$ & $\begin{array}{c}\text { HER2- } \\
\text { positive }(n=46)\end{array}$ & $\begin{array}{c}\text { HER2- } \\
\text { positive and HR- } \\
\text { positive }(n=25)\end{array}$ & $\begin{array}{l}\text { HER2- } \\
\text { positive and HR- } \\
\text { negative }(n=21)\end{array}$ & $\begin{array}{l}\text { HR-positive and } \\
\text { HER2- negative } \\
(n=54)\end{array}$ \\
\hline $\begin{array}{l}\text { Clinical complete } \\
\text { response rate (CR) }\end{array}$ & $34(26.6 \%)$ & $8(28.6 \%)$ & $16(34.8 \%)$ & $7(28.0 \%)$ & $9(42.9 \%)$ & $10(18.5 \%)$ \\
\hline $\begin{array}{l}\text { Clinical partial } \\
\text { response rate (PR) }\end{array}$ & $78(60.9 \%)$ & $15(53.5 \%)$ & $26(56.5 \%)$ & $17(68.0 \%)$ & $9(42.9 \%)$ & $37(68.5 \%)$ \\
\hline $\begin{array}{c}\text { Objective } \\
\text { responses rate } \\
\text { (ORR) }\end{array}$ & $112(87.5 \%)$ & $23(82.1 \%)$ & $42(91.3 \%)$ & $24(96 \%)$ & $18(85.7 \%)$ & $47(87.0 \%)$ \\
\hline $\begin{array}{l}\text { Progressive } \\
\text { Disease (PD) }\end{array}$ & $4(3.1 \%)$ & $1(3.6 \%)$ & $2(4.3 \%)$ & 0 & $2(9.5 \%)$ & $1(1.9 \%)$ \\
\hline
\end{tabular}

pathology type such as squamous subtype or micropapillary carcinoma or with neuroendocrine differentiation). 3 patients receive upfront surgery and another 1 patient progressed after the last cycle of NACT. Although they all received radical surgery due to close follow-up during NACT, 50\% (2/4) patients died from tumor progression afterwards. 


\section{Discussion}

There was a high compliance of neoadjuvant chemotherapy (NACT) with support of Multidisciplinary Team (MDT) in our real-world practice in breast cancer. The reasons of refusing NACT were mainly worrying about delaying surgery or toxicity of chemotherapy, and patient preference of Chinese traditional medicine therapy. 3 year EFS of patients who refused NACT was much worse than those received NACT. 89.1\% patients had completed all planned NACT and reasons of not completing all cycles were suboptimal clinical response to treatment, side effects and patient preference. Patients with subtypes of HER2-positive and triple negative breast cancer, ki67 $\geq 30 \%$ trended to have higher pCR. At median follow-up time of $40.0 \mathrm{~m}, 3$ year EFS was better in pCR patients than in non-pCR patients. With the support of MDT in southern China, the compliance rate of NACT was higher than that of another real world study in Australia (68.4\%) [19]. Our study found reasons of refusing NACT were mainly worried about delayed surgery or side effects of chemotherapy, which was consistent with the cross-sectional survey of Zdenkowski $\mathrm{N}$ et al. [20]. In their study, the main barriers of NACT were patients' demand of surgery as soon as possible, lack of proper understanding of NACT, fear of disease progression, and lack of interest in the downtime [20]. 3 year EFS of patients who hadn't accept NACT was much worse than those received NACT, especially patients who chose Chinese traditional medicine therapy and worried about toxicity of chemotherapy.

Although the benefit of compliance with NACT in breast cancer has not been previously reported in large study, 5-year local recurrence rate doubled in patients who didn't accept chemotherapy in a study of rectal cancer compared with those accepted chemotherapy [21]. Myth with traditional Chinese medicine was another barrier of NACT in Chinese patients as 3 patients refused the recommendation of NACT in this study and all of them progressed to stage IV diseases. Therefore, close follow-up, better education of patients and team cooperation of managing patients are important for NACT. It was also suggested that adjuvant information should be provided to patients during the visit of the surgeon and oncologist to facilitate the decision to receive NACT [22] and eliminate their worries about chemotherapy in time. The most important of all, NACT is recommended to be managed by Multidisciplinary Team (MDT) for better monitoring tumor and managing patients by team cooperation. Reasons of not completing all planned NACT were suboptimal clinical response to treatment, side effects and patient preference. We can give proper preventive intervention for patients who planned to receive highly toxic chemotherapeutic regimens before NACT to improve the rate of patients completing all planned chemotherapy cycles. Patients with suboptimal clinical response to NACT were mainly special pathology types (such as squamous subtype or micropapillary carcinoma or with neuroendocrine differentiation). $4 / 128$ patients progressed during NACT and $50 \%$ of them died afterwards due to tumor progression, in spite of they all received subsequent radical surgery after NACT.

We could give intense individualized therapy for this group of patients with high risk pathology types to improve their survival. Closer clinical monitoring including imagine (especially MR) is needed during NACT to avoid delayed detection of tumor progression during treatment and avoid losing the possibility of radical surgery [23]. It seems MDT is the best way so far to get the support of radiologists as a team member to better monitor tumor and improve care of patients. As for outcomes of the 128 patients who had accepted NACT and received surgery subsequently, pathology complete response rate was higher in more aggressive subtypes (triple negative and HER2-positive breast cancer), which was consistent with results of several studies [7,9]. Since pertuzumab is unavailable in mainland China before 2019, most HER2-positive patients received NACT of docetaxel/carboplatin/ trastuzumab with pCR rate of $32.6 \%$, which was non-inferiority to that of other 2 studies $(29.0 \%$ in NeoSphere study [15] and $21.8 \%$ in PEONY study of Asia patients [14]). We believe pCR rate of this subtype could be improved in future with the combination of pertuzumab since the total pCR rate could rise to $39.3 \%$ in pertuzumab /trastuzumab /docetaxel group in Chinese patients [14]. Among HER2-positive breast cancer, pCR rate of HR-negative subgroup was the highest, which was consistent with the results of other studies [14,15,24-26].

pCR for triple negative breast cancer was $32.1 \%$ in this study while it ranged from $22 \%$ to $52.1 \%$ in clinic trails $[10,27,28]$. The rate of $\mathrm{pCR}$ in patients with HR-positive/HER2-negative breast cancer was the lowest of all subtypes, which was similar with the result (7.5-16.2\%) of a pooled analysis [7]. 3 year EFS in this study trended to be better in patients with pCR than those with non-pCR, which was consistent with results of several studies [7-10]. Since patients with optimal response to NACT had better compliance and improved survival benefits, NACT is more recommended for patients with triple negative and HER2- positive breast cancer, or those with ki67 $\geq 30 \%$, except for the need of BCT or better surgery in some local advanced HR-positive/HER2-negative breast cancer. The limitation of the study includes the nature of a retrospective study, presence of confounding factors and lower compliance rate than rigorously designed clinical trials. However, the study carries significant findings as it can reflect regional real world practice and every patient was regularly recorded after MDT. Secondly, survival data was not mature in this study with inadequate study population. Further research of larger sample and longer followup is needed to validate our findings, help us better perform neoadjuvant chemotherapy and improve long term survival of breast cancer patients. 


\section{Conclusion}

There was a high compliance rate of neoadjuvant chemotherapy (NACT) with the support of MDT in Southern China. NACT should be managed by MDT to improve both treatment compliance and quality. NACT is more favorable for patients with triple negative and HER2-positive breast cancer or with higher ki67 since they had higher pathological complete response rates, compared to HRpositive/HER2-negative breast cancer.

\section{Conflict of Interest}

The authors declare no potential conflict of interest.

\section{Funding Statement}

This project was supported by Shenzhen Key Medical Discipline Construction Fund (No. SZXK014), Health Commission of Guangdong Province, China [A2021114], Shenzhen Fundamental Research Program (No. JCYJ2020109150427184).

\section{Data Availability Statement for this Work}

The datasets analyzed during the current study are not publicly available due to privacy but are available from the corresponding author on reasonable request.

\section{Authors' Contribution}

a. Fang Chen: Primary investigator: overall study hypothesis, study design, data collection, data analysis, result interpretation, manuscript writing and final manuscript approval.

b. Lingyu Ma: Various parts of statistical analysis, results interpretation and final manuscript approval.

c. Fan Yi, Manling Zhou, Lin Shen, Yaya Liu: Clinic data collection and final manuscript approval.

d. Haiman Jing, Jian Shi, Amy Chang and Ka On Lam: Results interpretation and final manuscript approval.

e. Hao Yu: data quality control and final manuscript approval

\section{References}

1. Mauri D, Pavlidis N, Ioannidis JP (2005) Neoadjuvant versus adjuvant systemic treatment in breast cancer: a meta-analysis. Journal of the National Cancer Institute 97(3): 188-194.

2. Kaufmann M, Von Minckwitz G, Mamounas EP, Cameron D, Carey LA, et al (2012) Recommendations from an international consensus conference on the current status and future of neoadjuvant systemic therapy in primary breast cancer. Annals of surgical oncology 19(5): 1508-1516.

3. Kümmel S, Holtschmidt J, Loibl S (2014) Surgical treatment of primary breast cancer in the neoadjuvant setting. British Journal of Surgery 101(8): 912-924

4. Gralow JR, Burstein HJ, Wood W, Hortobagyi GN, Gianni L, et al. (2008) Preoperative therapy in invasive breast cancer: pathologic assessment and systemic therapy issues in operable disease. Journal of Clinical Oncology 26(5): 814-819.
5. Hagerty RG, Butow PN, Ellis PM, Lobb EA, Pendlebury SC, et al. (2005) Communicating with realism and hope: incurable cancer patients' views on the disclosure of prognosis. Journal of clinical oncology 23(6): 12781288.

6. Schofield PE, Butow PN, Thompson JF, Tattersall MHN, Beeney LJ, et al. (2003) Psychological responses of patients receiving a diagnosis of cancer. Annals of oncology 14(1): 48-56.

7. Von Minckwitz G, Untch M, Blohmer JW, Costa SD, Eidtmann, et al. (2012) Definition and impact of pathologic complete response on prognosis after neoadjuvant chemotherapy in various intrinsic breast cancer subtypes. J Clin oncol 30(15): 1796-1804.

8. Von Minckwitz G, Untch M, Nuesch E, Loibl S, Kaufmann M, et al. (2011) Impact of treatment characteristics on response of different breast cancer phenotypes: pooled analysis of the German neo- adjuvant chemotherapy trials. Breast cancer research and treatment 125(1): 145156.

9. Cortazar P, Zhang L, Untch M, Mehta K, Costantino P, et al. (2014) Pathological complete response and long-term clinical benefit in breast cancer: the CTNeoBC pooled analysis. The Lancet 384(9938): 164-172.

10. Liedtke C, Mazouni C, Hess KR, Andre F, Tordai A, et al. (2008) Response to neoadjuvant therapy and long-term survival in patients with triplenegative breast cancer. Journal of clinical oncology 26(8): 1275-1281.

11. Symmans WF, Peintinger F, Hatzis C, Rajan R, Kuerer H, et al. (2007) Measurement of residual breast cancer burden to predict survival after neoadjuvant chemotherapy. Journal of Clinical Oncology 25(28): 44144422 .

12. Von Minckwitz G, Huang CS, Mano MS, Loibl S, Mamounas EP, et al. (2019) Trastuzumab emtansine for residual invasive HER2- positive breast cancer. New England Journal of Medicine 380(7): 617-628.

13. Masuda N, Lee SJ, Ohtani S, Im YH, Lee ES, et al. (2017) Adjuvant capecitabine for breast cancer after preoperative chemotherapy. New England Journal of Medicine 376(22): 2147- 2159.

14. Shao Z, Pang D, Yang H, Li W, Wang S, et al. (2020) Efficacy, safety, and tolerability of pertuzumab, trastuzumab, and docetaxel for patients with early or locally advanced ERBB2-positive breast cancer in Asia: the PEONY phase 3 randomized clinical trial. JAMA oncology 6(3): e193692.

15. Gianni L, Pienkowski T, Im YH, Roman L, Tseng LM, et al. (2012) Efficacy and safety of neoadjuvant pertuzumab and trastuzumab in women with locally advanced, inflammatory, or early HER2- positive breast cancer (NeoSphere): a randomised multicentre, open-label, phase 2 trial. The lancet oncology 13(1): 25-32.

16. Hurvitz SA, Martin M, Symmans WF, Jung KH, Huang CS, et al. (2018) Neoadjuvant trastuzumab, pertuzumab, and chemotherapy versus trastuzumab emtansine plus pertuzumab in patient with HER2-positive breast cancer (KRISTINE): a randomised, open-label, multicentre, phase 3 trial. The Lancet Oncology 19(1): 115-126.

17. Landercasper J, Dietrich LL, Johnson JM (2006) A breast center review of compliance with National Comprehensive Cancer Network Breast Cancer guidelines. The American journal of surgery 192(4): 525-527.

18. Komenaka IK, Pennington RE, Schneider BP, Hsu CH, Norton LE, et al. (2010) Compliance differences between patients with breast cancer in university and county hospitals. Clinical breast cancer 10(5): 385-391.

19.Zdenkowski N, Butow PN, Fewster S, Beckmore C, Wells K, et al. (2014) Exploring decision making about neoadjuvant chemotherapy for early breast cancer. American Society of Clinical Oncology 32: e20578.

20.Zdenkowski N, Butow P, Mann B, Fewster S, Douglas C, et al. (2015) Decisions about neoadjuvant systemic therapy for breast cancer: a survey of Australian and New Zealand specialists. ANZ journal of surgery 85(11): 797-798. 
21. Bosset JF, Collette L, Calais G, Mineur L, Maingon P, et al. (2006) Chemotherapy with preoperative radiotherapy in rectal cancer. New England Journal of Medicine 355(11): 1114-1123.

22. Herrmann A, Boyle F, Butow P, Hall AE, Zdenkowski N, et al. (2018) Exploring women's experiences with a decision aid for neoadjuvant systemic therapy for operable breast cancer. Health science reports 1(1): e13.

23. Gianni L, Eiermann W, Semiglazov V, Manikhas A, Lluch A, et al. (2010) Neoadjuvant chemotherapy with trastuzumab followed by adjuvant trastuzumab versus neoadjuvant chemotherapy alone, in patients with HER2-positive locally advanced breast cancer (the NOAH trial): a randomised controlled superiority trial with a parallel HER2-negative cohort. The Lancet 375(9712): 377-384

24. Schneeweiss A, Chia S, Hickish T, Harvey V, Eniu A, et al. (2013) Pertuzumab plus trastuzumab in combination with standard neoadjuvant anthracycline-containing and anthracycline-free chemotherapy regimens in patients with HER2-positive early breast cancer: a randomized phase II cardiac safety study (TRYPHAENA). Annals of oncology 24(9): 2278-2284.

\section{ISSN: 2574-1241}

DOI: $10.26717 /$ BJSTR.2021.36.005806

Hao Yu. Biomed J Sci \& Tech Res

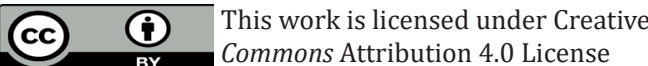

Submission Link: https://biomedres.us/submit-manuscript.php
25. Swain S, Ewer MS, Viale G, Delaloge S, Ferrero JM, et al. (2018) Pertuzumab, trastuzumab, and standard anthracycline-and taxanebased chemotherapy for the neoadjuvant treatment of patients with HER2-positive localized breast cancer (BERENICE): a phase II, openlabel, multicenter, multinational cardiac safety study. Annals of Oncology 29(3): 646-653.

26. Van Ramshorst MS, Van der Voort A, Van Werkhoven ED, Mandjes IA, Kemper I, et al. (2018) Neoadjuvant chemotherapy with or without anthracyclines in the presence of dual HER2 blockade for HER2-positive breast cancer (TRAIN-2): a multicentre, open-label, randomised, phase 3 trial. The lancet oncology 19(12): 1630-1640.

27. Schmid P, Cortes J, Pusztai L, McArthur H, Kummel S, et al. (2020) Pembrolizumab for early triple-negative breast cancer. New England Journal of Medicine 382(9): 810-821.

28. Poggio F, Bruzzone M, Ceppi M, Ponde NF, Valle GL, et al. (2018) Platinum-based neoadjuvant chemotherapy in triple- negative breast cancer: a systematic review and meta-analysis. Annals of oncology 29(7): 1497-1508.

\begin{tabular}{ll} 
BIOMEDICAL & Assets of Publishing with us \\
\hline RESEARCHES & - Global archiving of articles \\
\hline ISS: $2574-1241$ & - Immediate, unrestricted online access \\
\hline
\end{tabular}

